\title{
Diffraction-free pulses at arbitrary speeds
}

\author{
Janne Salo and Martti M Salomaa \\ Helsinki University of Technology, Materials Physics Laboratory, \\ PO Box 2200, FIN-02015 HUT, Finland \\ E-mail: Janne.Salo@hut.fi \\ Received 20 April 2001, in final form 13 June 2001 \\ Published 23 July 2001 \\ Online at stacks.iop.org/JOptA/3/366
}

\begin{abstract}
We consider periodically propagating pulses, devoid of diffractive

spreading. They may feature arbitrary velocities of propagation but their spectral characteristics vary according to whether they are luminal,

subluminal or superluminal. The wave modes introduced are closely related

to the $\mathrm{X}$ waves and the focus wave modes, but they allow a

frequency-dependent cone angle and are not limited to the speed of light.
\end{abstract}

Keywords: Non-diffracting waves, Bessel beams, X waves, superluminal

propagation, localized waves

\section{Introduction}

Recently, localized wave propagation has attracted much attention, and several propagation-invariant wave modes have been presented, such as focus wave modes $[1,2]$, $\mathrm{X}$ waves $[3,4]$ and subsonic nondiffracting waves (NDW) [5]. Here we introduce a systematic approach to all periodically evolving pulsed wave modes whose core propagates at an arbitrary velocity $0<v<\infty$. The spectral characteristics of the wave modes vary according to whether the velocity of propagation equals, exceeds, or is below the speed of light.

The propagating localized waves presented here share many properties of the $\mathrm{X}$ waves and the focus wave modes. Lateral localization is due to radial energy propagation which focuses on the axis; the characteristic $r^{-1}$ decay in energy density owes to this effect. The periodicity of the wave modes also leads to a 'carrier wave' whose velocity is $c^{2} / v$. Hence the latter is superluminal for pulses propagating with $v<c$, and subluminal for $v>c$. In particular, for $v=c$ the carrier wave propagates in the opposite direction, in analogy with the focus wave modes.

Requirements for the periodic propagation of the wave pulse are independent of the scalar or vector nature of the field. Essentially, the field decomposition is based on Bessel beams whose axial and radial wavenumbers scale with frequency. The vector description of the electromagnetic field is directly obtained by substitution of vector Bessel beams. Depending on the choice of the individual beam polarizations, different modes are obtained for the propagating pulses.

Although the detailed modulation of optical pulses in the inverse-frequency timescale is complicated, approximately propagation-invariant waves have been produced and observed [6]. The periodically evolving wave modes presented here allow for improved control over the velocity of pulse propagation. Moreover, they also provide new insight into the properties of classical superluminal electromagnetic waves [7].

We first consider the mathematical prerequisites for the discussion of periodically propagating wave fields and show how the different known wave modes, e.g. NDW and selfimaging fields, are related to the present formulation. We then briefly concentrate on monochromatic waves and subsequently focus on separately analysing the different velocity ranges. We also develop explicit integral expressions for all the singlemode waves which feature harmonic (sinusoidal) evolution under propagation.

\section{Periodic wave modes}

In this section, we discuss solutions of the scalar wave equation which 'retain' their spatial shape under propagation, i.e. which do not spread, or if they do, they will spontaneously return to their original form. We call these waves rotationally periodic waves (RPW); their mathematical definition is given below.

Consider an arbitrary field $\Phi$ which propagates along $z$, and express it in terms of spatial cylindrical coordinates, $(r, \varphi, z)$, and time, $t$. We require the field to be periodic in the following sense:

$$
\begin{aligned}
\Phi(r, \varphi+\gamma, z+\xi ; t+\tau) & =\Phi(r, \varphi+\gamma, z+v \tau ; t+\tau) \\
& =\Phi(r, \varphi, z ; t) .
\end{aligned}
$$

Furthermore, the field $\Phi$ is also periodic in the azimuthal angle $\varphi$ :

$$
\Phi(r, \varphi+2 \pi, z ; t)=\Phi(r, \varphi, z ; t) .
$$


Diffraction-free pulses at arbitrary speeds

The first condition may be interpreted as follows: after a certain period of time $\tau$, the field has propagated the distance $\xi=v \tau$ along $z$, and it has simultaneously rotated through the angle $\gamma$. We refer to $v$ as the velocity of propagation. We point out that no assumptions have been introduced concerning the behaviour of the field between the instants $t$ and $t+\tau$; i.e. it is not assumed to rotate uniformly but it may rather evolve in an arbitrarily complicated manner. Similar wave solutions have also been studied by Piestun and Shamir in [8], but we take a more general starting point and we shall obtain the monochromatic waves as a special case.

These two periodicity requirements lead to the RPW dispersion relation (see the appendix for a detailed derivation):

$$
k_{z, l n}=\frac{\omega}{v}+\frac{2 \pi l-n \gamma}{\xi} \equiv \frac{\omega}{v}+\mu,
$$

where $l$ and $n$ are arbitrary integers.

The field is also required to satisfy the scalar wave equation

$$
\nabla^{2} \Phi=\frac{1}{c^{2}} \frac{\partial^{2}}{\partial t^{2}} \Phi
$$

In the Fourier space, the wave equation reduces simply to $k_{x}^{2}+k_{y}^{2}+k_{z}^{2}=k_{\perp}^{2}+k_{z}^{2}=\omega^{2} / c^{2}$, where the phase velocity of isotropic wave motion is denoted by $c$ - to be distinguished from the propagation velocity, $v$. It immediately follows that for a given $\omega$ and $k_{z}$, the radial wavenumber $k_{\perp}$ is uniquely determined and it is given by

$$
k_{\perp, \ln }=\left(\frac{\omega^{2}}{c^{2}}-k_{z, l n}^{2}\right)^{1 / 2} .
$$

Note that the radial wavenumber only has an absolute value for waves which are defined on the axis of propagation ${ }^{1}$. Combining the conditions for the axial and radial wavenumbers and performing the inverse Fourier transform leads to

$$
\begin{gathered}
\Phi(r, \varphi, z ; t)=\sum_{l, n} \exp \{\mathrm{i}[n \varphi+(2 \pi l-\gamma n) z / \xi]\} \\
\quad \times \int a_{l n}(\omega) J_{n}\left(r k_{\perp, l n}\right) \exp [\mathrm{i} \omega(z / v-t)] \mathrm{d} \omega,
\end{gathered}
$$

which is the general expression for rotationally periodic solutions to the scalar wave equation. Here the $a_{l n}$ are arbitrary functions of frequency $\omega$. Each integral is evaluated over those frequencies for which the radial wavenumber possesses a positive real value.

The integral is a 'wave' that depends only on $r$ and $z-v t$ and, therefore, it propagates with the velocity $v$. The actual solution is a sum of such waves, modulated by exponentials that fulfil the periodicity requirements.

Finally, we want to emphasize the differences between the periodicities arising from the $l$ and $n$ degrees of freedom. The different $n$ define the azimuthal dependence of the wave for fixed $z$ and $t$. Furthermore, it is also guaranteed that this azimuthal shape is repeated after the pitch $\xi$ and the period $\tau$, only rotated through the angle $\gamma$. The mode $l=0$ represents uniform evolution of the wave while higher modes will change the shape of the wave within the period.

1 Hankel-based waves would also feature a radial direction but they contain a source along $z$. For Hankel waves, see, e.g., [4,9].
Table 1. Classification of different RPW. If $n$ is bound to zero, the rotational parameter $\gamma$ plays no role. Therefore, it is omitted from the rotationally invariant waves.

\begin{tabular}{llll}
\hline Wave type & $\gamma$ & $n$ & $l$ \\
\hline General RPWs & free & free & free \\
Uniformly propagating waves & free & free & $l=0$ \\
Nondiffracting waves & $\gamma=0$ & free & $l=0$ \\
Self-imaging waves & $\gamma=0$ & free & free \\
Rotationally invariant waves & - & $n=0$ & free \\
\hline
\end{tabular}

\section{Special propagation symmetries}

Several special cases of RPWs are of intrinsic interest in their own right. Hence we briefly describe some of them. Different subclasses are summarized in table 1 .

\subsection{Uniformly propagating waves}

General RPWs may display complicated behaviour within each period. Therefore, we first consider the case of uniformly propagating waves which have trajectories of constant value under propagation. That is, we require that the periodicity condition holds for all

$$
\begin{aligned}
& \xi=v \tau \\
& \gamma=v \tau
\end{aligned} \quad \tau \in \mathbb{R}
$$

i.e. that the constant-value trajectories are given by $(r \cos (\varphi+$ $\nu \tau), r \sin (\varphi+v \tau), z+v \tau)$ for all $r, \varphi$, and $z$. We shall, however, obtain uniformly propagating waves by considering the case $l=0$ of the general RPWs. This can be deduced from equation (A.4), which in this case is independent of $\theta$ and, consequently, we may take $l=0$. On the other hand, if the spatial period $\xi$ is small, the higher components $l \neq 0$ can only exist for very high frequencies. Hence, if the frequency band is bounded from above, the higher components are never excited which results in uniformly propagating waves.

\subsection{Nondiffracting waves}

NDW are a widely studied class of wave solutions. They are defined as wave fields which propagate uniformly without rotation: $\Phi(r, \varphi, z, t)=\Phi(r, \varphi, z-v t)$. Clearly, they form a subclass of uniformly rotating waves, with a vanishing rotation angle, $\gamma=0$.

NDW are also often called ' $\mathrm{X}$ waves' due to their conical form [3], and those NDW with fixed $n \neq 0$ are referred to as 'rotating $\mathrm{X}$ waves', cf equation (12) in [3]. However, their rotation is of static nature and it is not involved in the propagation dynamics of the wave. This is due to the fact that $\gamma=0$.

\subsection{Self-imaging waves}

Self-imaging waves are nonrotating periodic waves which reproduce themselves with the period $\tau$ at $z+v \tau$, i.e. $\Phi(r, \varphi, z+$ $v \tau, t+\tau)=\Phi(r, \varphi, z, t)$. They have $\gamma=0$ but they may feature different $l$ and $n$ components. 

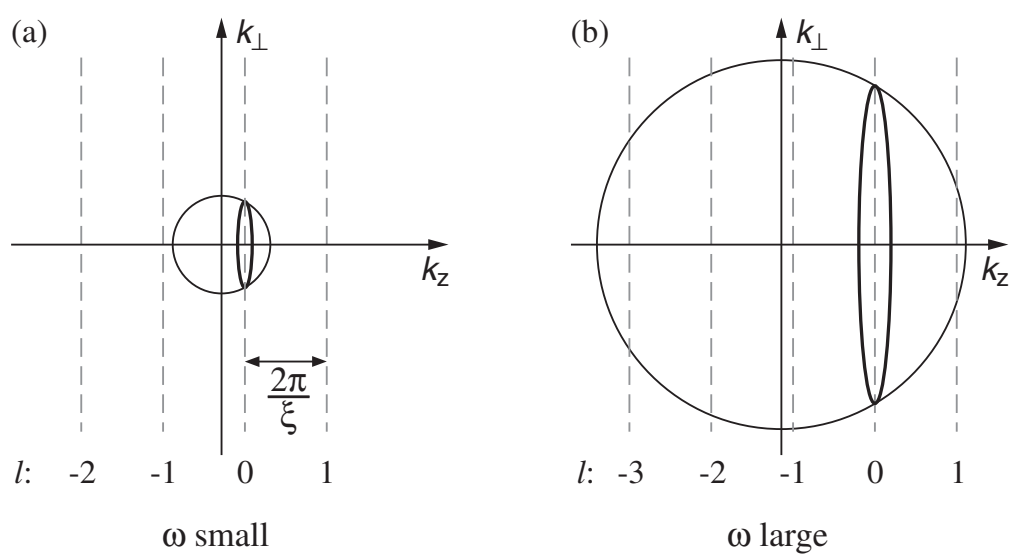

Figure 1. Permitted values of $k_{z}$ for a given $\omega$. The circle represents the dispersion relation $k_{\perp}^{2}+k_{z}^{2}=\omega^{2} / c^{2}$ which must be fulfilled by all the Fourier components of the wave. The fundamental mode $(n=l=0)$ is always possible, provided that $|v| \geqslant c$. The different $l$ modes share the frequency spacing of $2 \pi / \xi$ and, consequently, the higher modes can only exist if the frequency is high enough. In $(a)$, only the fundamental mode satisfies the dispersion relation, while in $(b)$ several higher modes are allowed. Both graphs are for the case with $v>c$ and $n=0$.

\subsection{Rotationally invariant waves}

Rotationally invariant waves are wave fields which have no azimuthal dependence. This leads directly to the condition $n=0$. On the other hand, while $n=0$, the wave becomes independent of the $\gamma$ parameter which may thus be set equal to zero. However, rotationally invariant waves may evolve during propagation, i.e. their cross section is not required to remain constant and, therefore, different $l$ modes are permitted which serves to make them a subclass of the self-imaging waves.

\section{Monochromatic fields}

We have found that the general expression for any RPW is given by a temporal inverse Fourier transform of

$$
\sum_{l} \sum_{n} a_{l n}(\omega) \exp \{\mathrm{i}[n \varphi+(2 \pi l-\gamma n) z / \xi]\} J_{n}\left(r k_{\perp, l n}\right) .
$$

Setting all $a_{l n}$ proportional to $\delta\left(\omega-\omega_{0}\right)$ (with a common $\omega_{0}$ for all $n$ and $l$ ), the monochromatic solution may be expressed in the form

$$
\begin{aligned}
& \Phi(r, \varphi, z ; t)=\sum_{l} \sum_{n} a_{l n} \exp \{\mathrm{i}[n \varphi+(2 \pi l-n \gamma) z / \xi]\} \\
& \quad \times J_{n}\left(r k_{\perp, l n}\right) \exp [\mathrm{i} \omega(z / v-t)],
\end{aligned}
$$

where the subscript 0 has been omitted from the $\omega$. Originally, both $l$ and $n$ were allowed to have any integer value. Here, however, the summation is much more limited. Since the $k_{z}$ spacings of the different $l$ and $n$ modes are $2 \pi / \xi$ and $\gamma / \xi$, respectively, only a finite number of modes can satisfy the dispersion relation $\left|k_{z}\right| \leqslant \omega / c$, see figure 1 . From the requirement that the radial wavenumber must be real and positive, we find that for a fixed frequency $\omega>0$ only such modes are permitted which fulfil the condition

$$
-\frac{v+c}{v c} \omega \leqslant \mu \leqslant \frac{v-c}{v c} \omega .
$$

This limitation also holds for polychromatic fields: when integrating over frequency, the Fourier weight of a given frequency must vanish for those $l$ and $n$ modes which are not permitted at that frequency. In particular, for $v \leqslant c$ the fundamental $\mu=0$ mode is not permitted since the upper bound is negative. For $v=c$, modes are available in the range

$$
-\frac{2}{c} \omega \leqslant \mu \leqslant 0
$$

Note that each term in equation (9) has the form of a Bessel beam

$$
\begin{aligned}
& \Phi(r, \varphi, z ; t)=\exp \{\mathrm{i}[n \varphi+(2 \pi l-n \gamma) z / \xi]\} \\
& \quad \times J_{n}\left(r k_{\perp, l n}\right) \exp [\mathrm{i} \omega(z / v-t)] \\
& =\exp (\mathrm{i} n \varphi) J_{n}\left(r k_{\perp, l n}\right) \exp \{\mathrm{i} \omega[(\mu / \omega+1 / v) z-t]\},
\end{aligned}
$$

which features the axial wavenumber $k_{z}=\omega / v+\mu$, as required by equation (3). We emphasize that the Bessel beam depends on $l$ and $\gamma$ only through the parameter $\mu$ which may be eliminated by further redefining the velocity of propagation as $v^{\prime}=(\mu / \omega+1 / v)^{-1}$. Individual Bessel beams thus have only two independent parameters, the velocity $v^{\prime}$ and the azimuthal order $n$. Since $k_{\perp}$ is real and positive,

$$
\left|k_{z}\right|=\left(\omega^{2} / c^{2}-k_{\perp}^{2}\right)^{1 / 2} \leqslant|\omega / c|,
$$

the (axial) phase velocity $v=\omega / k_{z}$ is necessarily superluminal for each Bessel beam ${ }^{2}$, independently of the parameters $v$ and $\mu$. It is only their superpositions (see equation (9)), or spectral generalizations (see below for single-mode waves) whose velocity of propagation is directly defined by the parameter $v$.

This ambiguity is a consequence of the definition given for the rotational periodicity of a wave field: we have required that the wave pattern is repeated after the time interval $\tau$ and spatial spacing $\xi$, which together define the velocity of propagation $v=\xi / \tau$ :

$$
\Phi(z+\xi, t+\tau)=\phi(z, t)
$$

for all $z$ and $t$. However, provided that the wave also satisfies

$$
\Phi(z+\xi, t+\tau / 2)=\phi(z, t)
$$

2 Bessel beams are conventionally defined within the concept of NDW, which always exhibit superluminal velocities of propagation. 
we may argue that the velocity of propagation is actually $v^{\prime}=2 \xi / \tau$, which is equally consistent. Therefore, the final velocity of propagation must be verified for each wave solution separately.

\section{Single-mode waves}

We have shown that all RPW can be decomposed into partial waves with fixed $l$ and $n$. In this section, we derive the general mathematical expressions for single-mode waves. All singlemode waves are expressed in the general form

$$
\begin{aligned}
& \Phi_{l n}(r, \varphi, z ; t) \\
& =\exp (\mathrm{in} \varphi) \int a(\omega) J_{n}\left(r k_{\perp}\right) \exp \left[\mathrm{i}\left(k_{z} z-\omega t\right)\right] \mathrm{d} \omega,
\end{aligned}
$$

where the $k_{z}$ and $k_{\perp}$ are given by equations (3) and (5), respectively. (We omit the subscripts $\ln$ here since they are fixed for single-mode waves.) However, the nature of such waves essentially depends on the velocity of propagation of the wave. One has to be careful in imposing the limits of integration since not all frequencies can support waves for a given $\mu$. The dispersion relation requires that $\left|k_{z}\right| \leqslant|\omega| / c$ or, equivalently, that $k_{\perp}$ be real. This leads to different frequency bands of superluminal, subluminal and luminal waves, and we derive expressions for each of these distinct cases separately.

The frequency band is further limited by the requirement of causality. Suppose that a pulse is generated on a planar aperture at $z=0$ and that it is to propagate in the direction of positive $z$. For isotropic wave motion, the group velocity of each plane-wave component points in the same (opposite) direction with the wavevector for positive (negative) frequency. Therefore, for $\omega>0$ the causality of pulse propagation requires $k_{z}>0$, and vice versa.

\subsection{Superluminal single-mode wave}

We now turn to consider a single-mode wave for fixed $l$ and $n$ (and $\mu$ ) for a superluminal propagation velocity $v>c$. We omit the case $v<-c$ which has exactly the same physical properties, apart from the direction of propagation, which is opposite.

Provided that $\mu=0, k_{z}$ is always less than $|\omega| / c$ and we may choose the limits of integration freely. This is, however, a special case which imposes that $2 \pi l=\gamma n$ and it can thus be satisfied only if $\gamma$ is a rational multiple of $\pi$. Although mathematically highly unlikely, this would often correspond to the experimental situation. On the other hand, the final solution becomes independent of both $\gamma$ and $l$, actually resulting in a NDW with the index $n$ (see section 3.2). Clearly, it will also satisfy the periodicity conditions specified by the values of $\gamma$, $l$, and $n$. The wave is then given by

$$
\begin{aligned}
& \Phi(r, \varphi, z ; t)=\exp (\mathrm{i} n \varphi) \int_{-\infty}^{\infty} a(\omega) J_{n}\left[r|\omega|\left(\frac{v^{2}-c^{2}}{v^{2} c^{2}}\right)^{1 / 2}\right] \\
& \quad \times \exp [\mathrm{i} \omega(z / v-t)] \mathrm{d} \omega,
\end{aligned}
$$

with $a(\omega)$ an arbitrary (integrable) function.

For $\mu \neq 0$, the nature of the superluminal wave mode changes essentially. The frequency band becomes restricted since, obviously, at low frequencies one cannot satisfy $\left|k_{z}\right| \approx$
Table 2. Frequency and wavenumber characteristics for superluminal single-mode waves.

\begin{tabular}{rlrl}
\hline \multicolumn{1}{c}{ Superluminal single-mode wave $(\mu \neq 0)$} \\
\hline Frequency band $\quad \omega$ & $\leqslant-|\mu| \frac{v c}{v+c}$ and \\
$\omega$ & $\geqslant|\mu| \frac{v c}{v-c} ;$ \\
$w$ & $<-1$ and $w>1$ \\
& Frequency $\quad$ & $=\frac{\mu v^{2} c}{v^{2}-c^{2}}\left(w+\frac{c}{v}\right)$ \\
& Axial wavenumber $\quad k_{z}$ & $=\frac{\omega}{v}+\mu$ \\
& $=\frac{\mu v c}{v^{2}-c^{2}} w+\frac{\mu v^{2}}{v^{2}-c^{2}}$ \\
& Radial wavenumber $\quad k_{\perp}$ & $=\sqrt{\frac{\omega^{2}}{c^{2}}-k_{z}^{2}}$ \\
& $=\left(\frac{\mu^{2} v^{2}}{v^{2}-c^{2}}\right)^{1 / 2} \sqrt{w^{2}-1}$
\end{tabular}

$|\mu|<|\omega| / c \rightarrow 0$. Change to a dimensionless variable of integration $w$ via the transformation

$$
\omega=\frac{\mu v^{2} c}{v^{2}-c^{2}}\left(w+\frac{c}{v}\right)
$$

yields

$$
\begin{aligned}
& \Phi(r, \varphi, z ; t)=\exp (\mathrm{in} \varphi) \exp \left[\mathrm{i} \mu \frac{v^{2}}{v^{2}-c^{2}}\left(z-\frac{c^{2}}{v} t\right)\right] \\
& \quad \times\left[\int_{-\infty}^{-1}+\int_{1}^{\infty}\right] a(w) J_{n}\left(r C \sqrt{w^{2}-1}\right) \\
& \quad \times \exp \left[\mathrm{i} \frac{\mu v^{2} c}{v^{2}-c^{2}}(z / v-t) w\right] \mathrm{d} w
\end{aligned}
$$

which serves to reveal the intrinsic structure of the superluminal wave modes $\left(C=\left[\mu^{2} v^{2} /\left(v^{2}-c^{2}\right)\right]^{1 / 2}\right.$, for the values of $\omega, k_{z}$ and $k_{\perp}$, see table 2). First of all, the integration range is divided into two disjoint parts, one containing negative frequencies (up to $-|\mu| v c /(v+c)$ ) and the other containing positive frequencies (starting from $|\mu| v c /(v-c)$ ). Secondly, the wave mode contains a propagating pulse core, described by the integral which is further modulated by a plane wave. Moreover, the modulating wave has the velocity $V=c^{2} / v$ and the characteristic frequency $\Omega=\mu v c^{2} /\left(v^{2}-c^{2}\right)$ which only depends on the velocity of propagation $v$ and the mode parameter $\mu$. The superluminality of the pulse is thus compensated by the correspondingly slower phase.

Finally, we point out that in the case of negative $\mu$ the direction of integration is reversed since now $\omega \propto-w$. This is seen in the exponential terms where $\mu$ still has a negative value, while $C$ only depends on the absolute value of $\mu$. A complex analytic expression for the pulse (that limited to $\omega>0$ ) is thus obtained in the integration range $w \geqslant 1$ for $\mu>0$ (and $w \leqslant-1$ for $\mu<0$ ), see figure 2 .

\subsection{Subluminal single-mode wave}

We now turn to consider subluminal single-mode waves which satisfy $0<v<c$. No fundamental $(\mu=0)$ mode exists for subluminal waves since $\left|k_{z}\right|=|\omega| / v \leqslant|\omega| / c$ cannot be satisfied for $v<c$ (this is why the NDW are always 


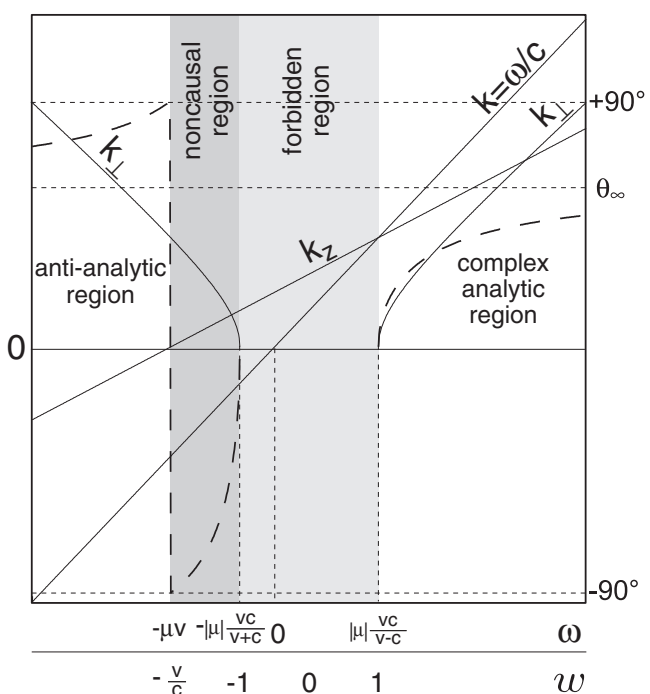

Figure 2. Scalar wavenumber $(k)$, and the axial $\left(k_{z}\right)$ and radial $\left(k_{\perp}\right)$ wavenumbers as functions of the dimensionless variable $w$ (for $\mu>0$ ). The dashed line shows the direction of wave propagation, measured in angles from the $z$ axis. For high $|\omega|$, the angle approaches the limit $\theta_{\infty}=\arccos (c / v)$. Within the noncausal region, the wave has a negative energy flux along $z$. The complex-analytic region is given by $\omega \geqslant 0$ while the anti-analytic region is defined by $\omega \leqslant 0$.

superluminal). For $\mu \neq 0$, the wave expression is obtained by a change of the frequency variable as

$$
\omega=\frac{\mu v^{2} c}{c^{2}-v^{2}}\left(w-\frac{c}{v}\right)
$$

and it is given by

$$
\begin{aligned}
& \Phi(r, \varphi, z ; t)=\exp (\mathrm{in} \varphi) \exp \left[-\mathrm{i} \mu \frac{v^{2}}{c^{2}-v^{2}}\left(z-\frac{c^{2}}{v} t\right)\right] \\
& \quad \times \int_{-1}^{1} a(w) J_{n}\left(r D \sqrt{1-w^{2}}\right) \\
& \quad \times \exp \left[\mathrm{i} \frac{\mu v^{2} c}{c^{2}-v^{2}}(z / v-t) w\right] \mathrm{d} w
\end{aligned}
$$

with $D=\left[\mu^{2} v^{2} /\left(c^{2}-v^{2}\right)\right]^{1 / 2}$, see table 3 . Therefore, subluminal waves are necessarily of limited bandwidth. Similarly to the superluminal case, the wave is composed of a propagating pulse and a phase, but here the velocity of the pulse is subluminal and that of the phase is superluminal. A subluminal wave is always anti-analytic $(\omega<0)$ for $\mu>0$ by necessity, and complex analytic $(\omega>0)$ for $\mu<0$, see figure 3 .

\subsection{Luminal single-mode waves}

Finally, consider the case of luminal waves with $v=c$. (We denote both velocities here with $c$.) The fundamental mode $\mu=0$ reduces to a pulse plane wave since $k_{\perp} \equiv 0$. For $\mu \neq 0$,

$$
\omega=-\frac{\mu c}{2}(w+1)
$$

yields

$\Phi(r, \varphi, z ; t)=\exp (\mathrm{in} \varphi) \exp \left[-\mathrm{i} \frac{\mu}{2}(z+c t)\right]$

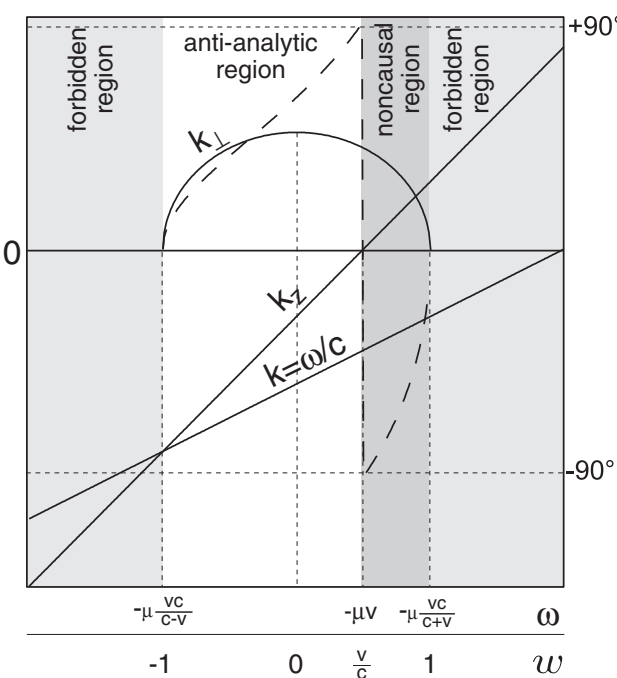

Figure 3. Scalar wavenumber $(k)$, and the axial $\left(k_{z}\right)$ and radial $\left(k_{\perp}\right)$

\begin{tabular}{|c|c|}
\hline \multicolumn{2}{|c|}{ Subluminal single-mode wave $(\mu \neq 0)$} \\
\hline Frequency band & $\begin{array}{l}-\mu \frac{v c}{c-v} \leqslant \omega \leqslant-\mu \frac{v c}{c+v}, \mu>0 \\
-\mu \frac{v c}{c+v} \leqslant \omega \leqslant-\mu \frac{v c}{c-v}, \mu<0 \\
-1 \leqslant w \leqslant 1\end{array}$ \\
\hline Frequency & $\omega=\frac{\mu v^{2} c}{c^{2}-v^{2}}\left(w-\frac{c}{v}\right)$ \\
\hline Axial wavenumber & $\begin{aligned} k_{z} & =\frac{\omega}{v}+\mu \\
& =\frac{\mu v c}{c^{2}-v^{2}} w-\frac{\mu v^{2}}{c^{2}-v^{2}}\end{aligned}$ \\
\hline Radial wavenumber & $\begin{aligned} k_{\perp} & =\sqrt{\frac{\omega^{2}}{c^{2}}-k_{z}^{2}} \\
& =\left(\frac{\mu^{2} v^{2}}{c^{2}-v^{2}}\right)^{1 / 2} \sqrt{1-w^{2}}\end{aligned}$ \\
\hline
\end{tabular}
wavenumbers as functions of the dimensionless variable $w$ (for $\mu>0$ ). No limiting cone angle exists for the subluminal waves.

Table 3. Frequency and wavenumber characteristics for subluminal single-mode waves.

$$
\times \int_{0}^{\infty} a(w) J_{n}(r|\mu| \sqrt{w}) \exp \left[-\mathrm{i} \frac{\mu}{2}(z-c t) w\right] \mathrm{d} w
$$

see table 4. Again, the wave consists of a propagating pulse and a modulating plane wave, both of which feature propagation at the speed of light. However, the modulation wave now propagates in the opposite direction in comparison to the pulse propagation. Similarly to subluminal waves, the luminal waves are anti-analytic for $\mu>0$ and analytic $(\omega>0)$ for $\mu<0$, see figure 4.

\section{Extension to vector fields}

Thus far, we have only considered the wave equation through its dispersion relation $k^{2}=\omega^{2} / c^{2}$ which is satisfied by all isotropic and dispersionless wave fields in homogeneous media. Diffraction-free scalar wave fields considered in this paper are composed of Bessel beams which explicitly satisfy 


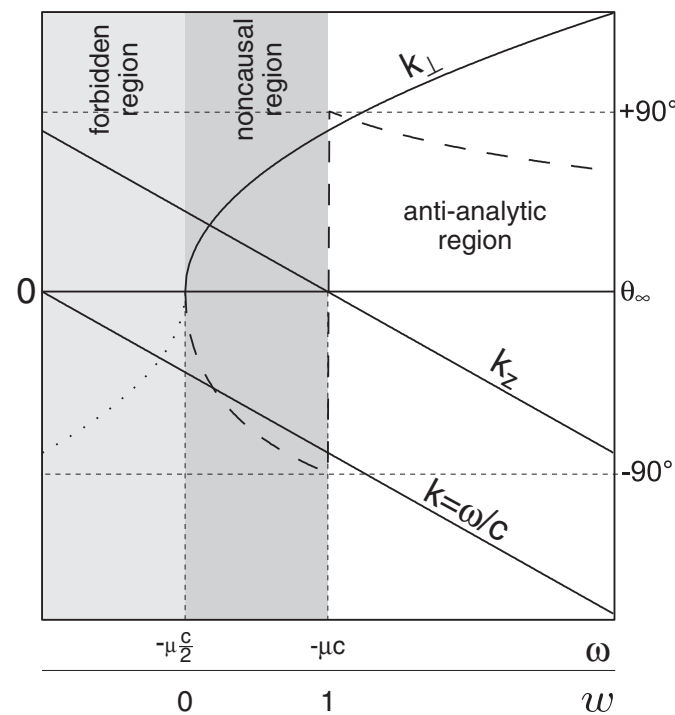

Figure 4. Scalar wavenumber $(k)$, and the axial $\left(k_{z}\right)$ and radial $\left(k_{\perp}\right)$ wavenumbers as functions of the dimensionless variable $w$ (for $\mu>0$ ). The limiting cone angle for luminal waves is zero, i.e. the high-frequency wave components propagate along $z$.

Table 4. Frequency and wavenumber characteristics for luminal single-mode waves.

\begin{tabular}{|c|c|}
\hline \multicolumn{2}{|c|}{ Luminal single-mode wave $(\mu \neq 0)$} \\
\hline Frequency band & $\begin{array}{l}\omega \leqslant-\mu \frac{c}{2}, \mu>0 \\
\omega \geqslant|\mu| \frac{c}{2}, \mu<0 ; \\
w \geqslant 0\end{array}$ \\
\hline Frequency & $\omega=-\frac{\mu c}{2}(w+1)$ \\
\hline Axial wavenumber & $\begin{aligned} k_{z} & =\frac{\omega}{c}+\mu \\
& =\frac{\mu}{2}(1-w)\end{aligned}$ \\
\hline Radial wavenumber & $\begin{aligned} k_{\perp} & =\sqrt{\frac{\omega^{2}}{c^{2}}-k_{z}^{2}} \\
& =|\mu| \sqrt{w}\end{aligned}$ \\
\hline
\end{tabular}

the following relation, see equation (6):

$$
\Phi_{J}(r, \varphi, z, t ; \omega)=\exp (\mathrm{in} \varphi) J_{n}\left(k_{\perp} r\right) \exp \left[\mathrm{i}\left(k_{z} z-\omega t\right)\right]
$$

Corresponding vector-valued fields are obtained by replacing the scalar Bessel beams with their vectorized counterparts (see, e.g., $[10,11])$. Using the plane-wave representation of Bessel beams, we find

$$
\begin{aligned}
& \Phi_{J}(r, \varphi, z, t ; \omega)=\frac{(-\mathrm{i})^{n}}{2 \pi} \int_{0}^{2 \pi} \exp (\mathrm{i} n \beta) \\
& \quad \times \exp \left[\mathrm{i}\left(x k_{\perp} \cos \beta+y k_{\perp} \sin \beta+z k_{z}-\omega t\right)\right] \mathrm{d} \beta .
\end{aligned}
$$

Vector beams are obtained simply by inserting a (unit) polarization vector $\boldsymbol{E}(\beta)$ which satisfies Maxwell's equations together with the above wavevector. In isotropic media, this is simply achieved by requiring that $\boldsymbol{E}(\beta) \perp \boldsymbol{k}(\beta)$ where $\beta$ is the azimuthal angle in the Fourier space, see figure 5. For a generalization to the case of anisotropic media, see [12].

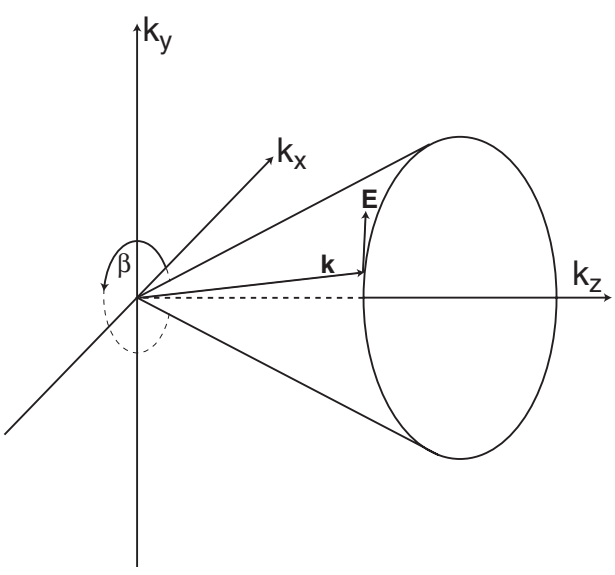

Figure 5. Wavevector representation of Bessel beams. The electric polarization $\boldsymbol{E}$ is orthonormal to the corresponding wavevector $\boldsymbol{k}$. Here $\beta$ is the azimuthal angle in the $\left(k_{x}, k_{y}\right)$ plane.

\section{Physical wave propagation}

Mathematically, no constraints were imposed on the shape of the propagating wave field. The requirement of periodic propagation, with or without rotation, leads to the axial dispersion relation for each mode, given by equation (3). However, the resultant fields, especially for single-mode waves, feature clear wavefront structure, see figure 6 . This is due to the single-valued dependence of both the axial and radial wavenumbers on frequency since, consequently, each frequency has a dominant direction of energy propagation, given by the group velocity.

A superluminal wave, figure $6(a)$, features the closest resemblance with the ordinary nondiffracting $X$ wave. The emerging central spot of the wave is constructed, at each instant of time, by the conically approaching wavefront which has been launched well before the formation of the current focal spot. The spatial dimensions of the pulse are determined by the radial wavenumber $C \sqrt{w^{2}-1}$ and the axial wavenumber $\left[\mu v c /\left(v^{2}-c^{2}\right)\right] w$, see equation (20). The precise form of the pulse is given by the spectral function $a(w)$. The modulating plane wave has $\Omega=\mu v c^{2} /\left(v^{2}-c^{2}\right), K_{z}=\mu v^{2} /\left(v^{2}-c^{2}\right)$, and $V=c^{2} / v$.

The wavefronts for a luminal field, figure $6(b)$, appear somewhat similar to those in superluminal waves and they are launched before the formation of the current spot but always appear equally far from the future one, causing it to move exactly with the speed of light. The dimension of the pulse owe to the radial and the axial wavenumbers, $|\mu| \sqrt{w}$ and $(\mu / 2) w$, respectively. The modulating plane wave has characteristic parameters given by $\Omega=\mu c / 2, K_{z}=-\mu / 2$ and $V=-c$. Note that in this particular case, the plane wave propagates in the opposite sense with respect to the pulse itself.

For subluminal waves, figure 6(c), the conical wavefronts precede the present focus of the wave, making the apparent pulse propagation slower than light. Note that the wavefront exhibits no prominent propagation angle but is nearly circular. The characteristic dimensions of the pulse are given by radial wavenumber, $D \sqrt{1-w^{2}}$, and by the axial wavenumber, $\mu v c /\left(c^{2}-v^{2}\right) w$, see equation (22). The plane wave features $\Omega=-\mu v c^{2} /\left(c^{2}-v^{2}\right), K_{z}=-\mu v^{2} /\left(c^{2}-v^{2}\right)$ and $V=c^{2} / v$. 

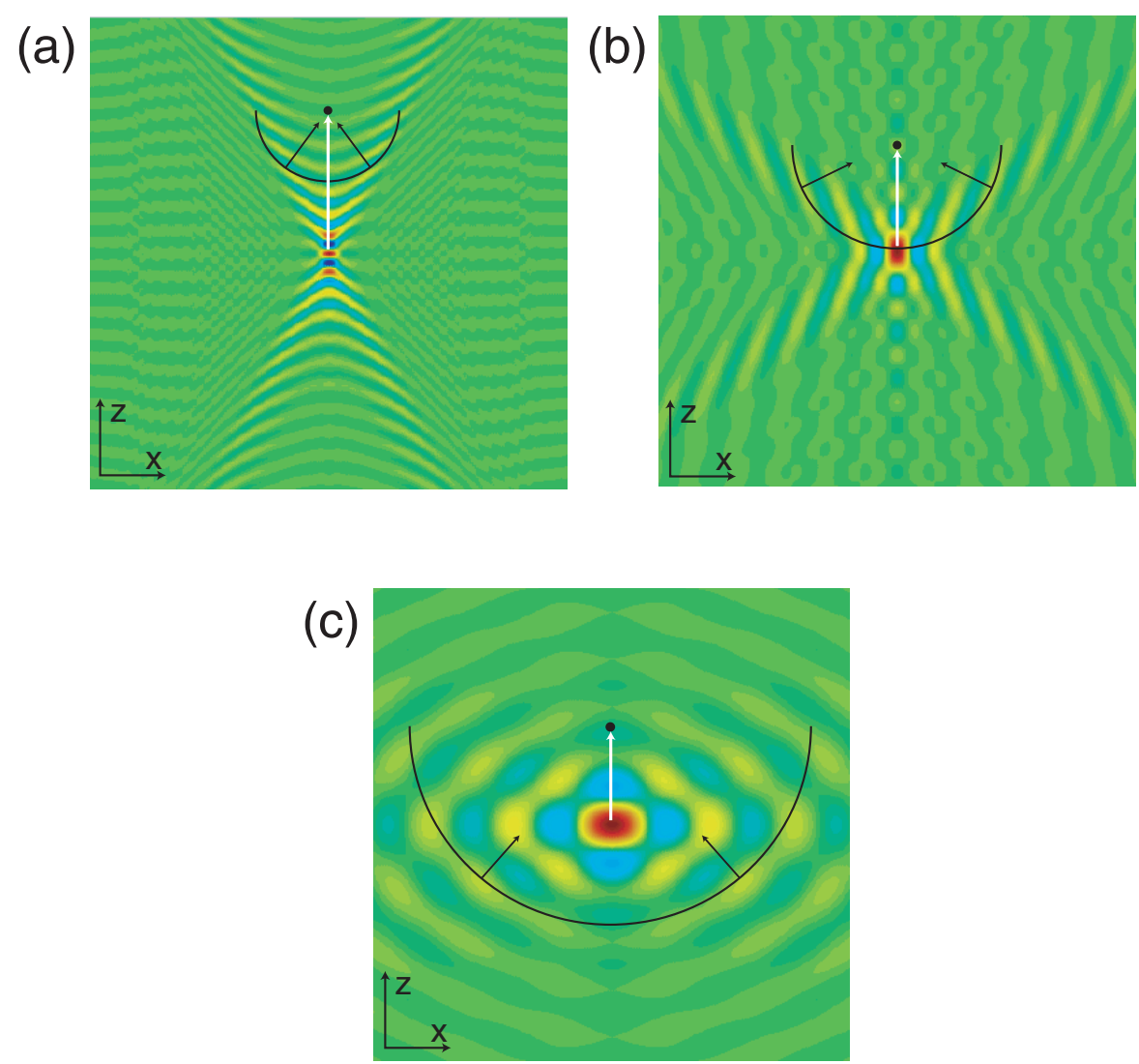

Figure 6. (a) Superluminal single-mode field for $v=2 c,(b)$ luminal field, and $(c)$ subluminal field for $v=c / 2$. The black arrows indicate the direction of energy propagation for wavefronts while the white arrows denote the propagation of the pulse centre. The length of the white arrow is proportional to the velocity of propagation, $v$, while the radius of the wavefront is proportional to the speed of light. (Black arrows are not to scale.)

\section{Discussion}

Diffraction-free and propagation-invariant wave fields have attracted intensive attention during the past decade. In this paper, we have presented a systematic formulation for periodically evolving localized pulses which may propagate at arbitrary speeds, in contrast to conventional $\mathrm{X}$ waves and focus wave modes.

Similar monochromatic wave fields have been widely studied, and the novel pulsed fields provide propagating counterparts to the NDW (Bessel beams), and the selfimaging and rotating fields. We have briefly considered the monochromatic solutions and then separately discussed singlemode waves for different velocity ranges. General integral expressions are obtained for all the modes. Finally, we have presented illustrations of the three simplest wave fields which demonstrate the characteristic properties of periodically evolving pulses.

We expect that the present theory of propagating wave fields will provide new insight and tools and thus contribute to the research of electromagnetic pulses.

\section{Acknowledgments}

JS is grateful to the Helsinki University of Technology, the Finnish Cultural Foundation, and the Jenny and Antti Wihuri Foundation for support. This research is also funded by the
Academy of Finland through the Research Programme on Electronic Materials and Microsystems (EMMA).

\section{Appendix. Dispersion relation for rotationally periodic waves}

Consider an arbitrary field which propagates along $z$ and express it using spatial cylindrical coordinates and time. We require the field to satisfy the periodicity conditions given by equations (1) and (2). The Hankel-Fourier transform of such a wave is given by

$$
\begin{aligned}
& \tilde{\Phi}\left(k_{\perp}, m, k_{z} ; \omega\right)=\frac{1}{2 \pi} \int \Phi(r, \varphi, z ; t) \exp (-\mathrm{i} m \varphi) J_{m}\left(k_{\perp} r\right) \\
& \quad \times \exp \left[-\mathrm{i}\left(k_{z} z-\omega t\right)\right] r \mathrm{~d} r \mathrm{~d} \varphi \mathrm{d} z \mathrm{~d} t .
\end{aligned}
$$

We change the variables of integration according to

$$
\begin{gathered}
z=\xi \eta+(\xi / 2 \pi) \theta \\
t=(\tau / 2 \pi) \theta \\
\varphi=(\gamma / 2 \pi) \theta+\alpha
\end{gathered}
$$

and obtain

$$
\begin{aligned}
& \tilde{\Phi}\left(k_{\perp}, m, k_{z} ; \omega\right) \\
& =\frac{v \tau^{2}}{(2 \pi)^{2}} \int \Phi(r, \gamma / 2 \pi \theta+\alpha, \xi \eta+\xi \theta / 2 \pi ; \tau \theta / 2 \pi) \\
& \quad \times \exp [-\mathrm{i} m(\gamma \theta / 2 \pi+\alpha)] J_{m}\left(k_{\perp} r\right)
\end{aligned}
$$


$\times \exp \left\{-\mathrm{i}\left[k_{x}(\xi \eta+\xi \theta / 2 \pi) z-\omega(\tau \theta / 2 \pi)\right]\right\} r \mathrm{~d} r \mathrm{~d} \alpha \mathrm{d} \eta \mathrm{d} \theta$,

where we employ cylindrical coordinates $k_{x}=k_{\perp} \cos \beta$ and $k_{y}=k_{\perp} \sin \beta$ in the Fourier space.

Now $\Phi$ has the period of $2 \pi$ for both $\alpha$ and $\theta$ and, therefore, we may write

$$
\Phi(r, \alpha, \eta, \theta)=\sum_{l=-\infty}^{\infty} \sum_{n=-\infty}^{\infty} a_{l n}(r, \eta) \exp [\mathrm{i}(n \alpha+l \theta)]
$$

Substitution into equation (A.3) yields

$$
\begin{gathered}
\tilde{\Phi}\left(k_{\perp}, m, k_{z} ; \omega\right)=\sum_{l=-\infty}^{\infty} \tilde{a}_{l m}\left(k_{\perp}, k_{z} \xi\right) \\
\times \delta\left[k_{z}-\left(\frac{\omega}{v}+\frac{2 \pi l-m \gamma}{\xi}\right)\right] .
\end{gathered}
$$

Since $a_{l n}(r, \eta)$ are arbitrary functions, their Fourier transforms, $\tilde{a}_{l m}$ are also arbitrary functions of $k_{\perp}$ and $k_{z} \xi$. As the deltafunction fixes $k_{z}$ for a given $\omega, l$, and $n$, we conclude that the integral may also be considered an arbitrary function of $k_{\perp}$ and $\omega$.

Now we demand that the ordinary scalar wave equation be obeyed. In the Fourier space, it reduces to $k_{x}^{2}+k_{y}^{2}+k_{z}^{2}=$ $k_{\perp}^{2}+k_{z}^{2}=\omega^{2} / c^{2}$ where the phase velocity for isotropic wave motion is denoted by $c$, in contrast to the propagation velocity $v$. It immediately follows that $k_{\perp}$ is uniquely determined for given $\omega$ and $k_{z}$; that is, for given $\omega, l$ and $n$. The Fourier representation may thus be expressed as

$$
\begin{gathered}
\tilde{\Phi}\left(k_{\perp}, m, k_{z} ; \omega\right)=\sum_{l} a_{l m}(\omega) \delta\left[k_{z}-\left(\frac{\omega}{v}+\frac{2 \pi l-m \gamma}{\xi}\right)\right] \\
\times \delta\left[k_{\perp}-\left(\frac{\omega^{2}}{c^{2}}-k_{z}^{2}\right)^{1 / 2}\right] .
\end{gathered}
$$

The field itself may now be represented in the form

$$
\begin{aligned}
& \Phi(r, \varphi, z ; t)=\sum_{n=-\infty}^{\infty} \sum_{l=-\infty}^{\infty} \int_{-\infty}^{\infty} a_{l n}(\omega) \\
& \quad \times \exp (\mathrm{i} n \varphi) J_{n}\left(k_{\perp, l n} r\right) \exp \left[\mathrm{i}\left(k_{z, l n} z-\omega t\right)\right] \mathrm{d} \omega
\end{aligned}
$$

where the factor $k_{\perp} / 2 \pi$ has been incorporated into the $a_{l n}(\omega)$. The axial wavenumber equals

$$
k_{z, l n}=\frac{\omega}{v}+\frac{2 \pi l-n \gamma}{\xi},
$$

while the radial wavenumber is

$$
k_{\perp, l n}=\left(\frac{\omega^{2}}{c^{2}}-k_{z, l n}^{2}\right)^{1 / 2} .
$$

In equation (A.7), it is required that the $a_{l n}(\omega)$ vanish for imaginary ${ }^{3} k_{\perp}$. The wave may also be represented as

$$
\begin{gathered}
\Phi(r, \varphi, z ; t)=\sum_{l} \sum_{n} \exp [\mathrm{i}(n \varphi+(2 \pi l-\gamma n) z / \xi)] \\
\quad \times \int_{-\infty}^{\infty} a_{l n}(\omega) J_{n}\left(r k_{\perp, l n}\right) \exp [\mathrm{i} \omega(z / v-t)] \mathrm{d} \omega .
\end{gathered}
$$

The integral is a 'wave' that only depends on $r$ and $z-v t$; therefore, it propagates with the velocity $v$. The actual solution is a sum of such waves, modulated by exponential terms that satisfy the periodicity requirements.

\section{References}

[1] Brittingham J N 1983 Focus wave modes in homogeneous Maxwell's equations: transverse electric mode J. Appl. Phys. 54 1179-89

[2] Reivelt K and Saari P 2000 Optical generation of focus wave modes J. Opt. Soc. Am. A 17 1785-90

[3] Lu J-y and Greenleaf J F 1992 Nondiffracting X waves-exact solutions to free-space scalar wave equation and their finite aperture realizations IEEE Trans. Ultrason. Ferroelectr. Freq. Control. 39 19-31

[4] Salo J, Fagerholm J, Friberg A T and Salomaa M M 2000 Unified description of nondiffracting $X$ and $Y$ waves Phys. Rev. E 62 4261-75

[5] Salo J and Salomaa M M 2001 Subsonic nondiffracting waves Acoust. Res. Lett. Online 2 31-6

[6] Saari P and Reivelt K 1997 Evidence of X-shaped propagation-invariant localized light waves Phys. Rev. Lett. 79 4135-8

[7] Mugnai D, Ranfagni A and Ruggeri R 2000 Observation of superluminal behaviors in wave propagation Phys. Rev. Lett. 84 4830-3

[8] Piestun R and Shamir J 1998 Generalized propagation-invariant wave fields J. Opt. Soc. Am. A 15 3039-44

[9] Chávez-Cerda S, McDonald G S and New G H C 1996 Nondiffracting beams: travelling, standing, rotating and spiral waves Opt. Commun. 123 225-33

[10] Mishra S R 1991 A vector wave analysis of a Bessel beam Opt. Commun. 85 159-61

[11] Hall D H 1996 Vector-beam solutions of Maxwell's wave equation Opt. Lett. 21 9-11

[12] Salo J, Fagerholm J, Friberg A T and Salomaa M M 1999 Nondiffracting bulk-acoustic X waves in crystals Phys. Rev. Lett. 83 1171-4

3 Otherwise, integration over $k_{\perp}$ yields zero since $\delta\left[k_{\perp}-\left(\omega^{2} / c^{2}-k_{z}^{2}\right)^{1 / 2}\right]=0$ for all $k_{\perp} \in \mathbb{R}$. 